Letter to Editor

\title{
Middle turbinate medialization: bolgerization or conchopexy suture
}

\section{Letter to editor}

The middle turbinate is a recognized key structure in Endoscopic Sinus Surgery and, an adequate management of it, remains somewhat controversial. ${ }^{1}$ Some authors opine that the middle turbinate should be resected, to avoid the common lateralization with the closure of the middle meatus and the failure of treatment (Figure 1A). However, others experts, prefer to conserve this structure in most of cases because of the functional importance of the middle turbinate in the humidifying inspired air, creating laminar airflow, ${ }^{2}$ and to avoid the loss of an important surgical landmark in revision endoscopic procedures. The management of this fragile structure should be carefully to avoid a cerebrospinal fluid leakage.

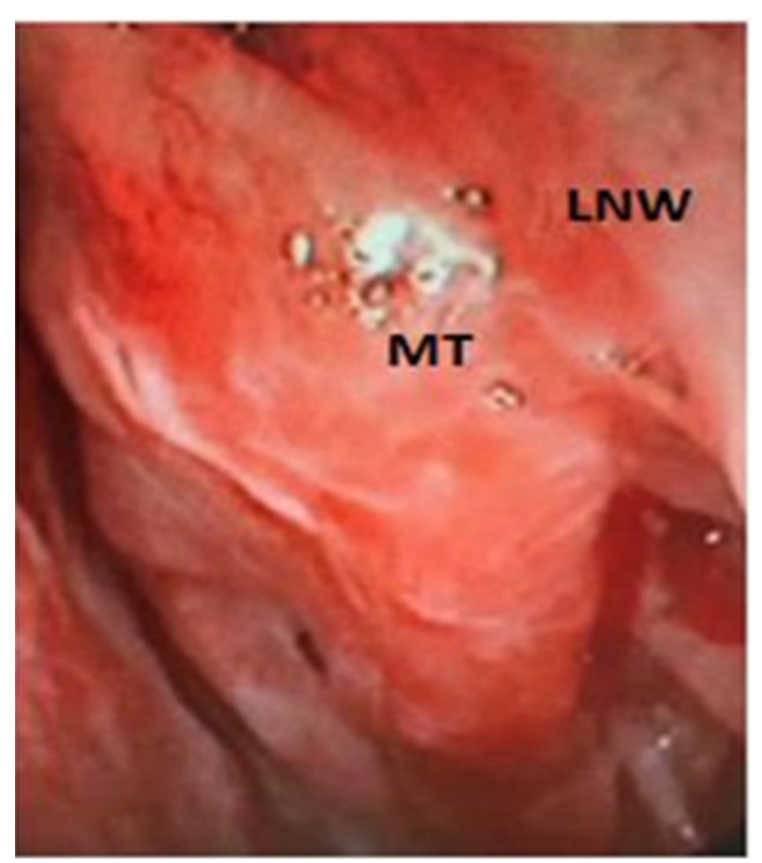

Figure IA Closure of the left middle meatus.

Several different techniques have been designed to keep the middle turbinate medialized, and to maintain the patency of the middle meatus that allows delivery of topical medication and sinus aeration (Figure 1B). These include middle meatal packing, stent or spacer placement, controlled synechiae formation between middle turbinate and the septum and suture medialization of the middle turbinate.

"Controlled Synechia" (Bolgerization) has been shown to be effective in prevention of lateralization in as many as $88 \%$ of patients ${ }^{3}$ (Figure 2). This technique was published by Bolger et al. in $1999^{4}$ and consists in the performance of mucosal abrasion on the medial aspect of the anteroinferior portion of the middle turbinate and, on the adjacent nasal septal mucosa using the sickle knife or the microdebrider. This technique requires postoperative nasal packing
Volume 4 Issue I - 2016

\author{
Pinilla Urraca Mayte \\ Department of Otorhinolaryngology, Puerta de Hierro \\ University Hospital, Spain
}

Correspondence: Mayte Pinilla Urraca, Department of Otorhinolaryngology Head \& Neck Surgery, C/ Joaquin Rodrigo, 2, 28002, Majadahonda, Madrid, Spain, Tel +346-298-10134, Email pinillamayte@gmail.com

Received: January 03, 2015 | Published: January 29, 2016

for up to 10days after surgery. The nasal packing is placed in the middle meatus to medialize the turbinate against the septum and join the incisions. Removable nasal packing is uncomfortable, produces local pressure and its removal can cause nasal bleeding and vasovagal reaction. Currently, biodegradable and absorbable materials are now routinely used after this surgery due to their fine hemostatic properties and to be more comfortable for the patient.

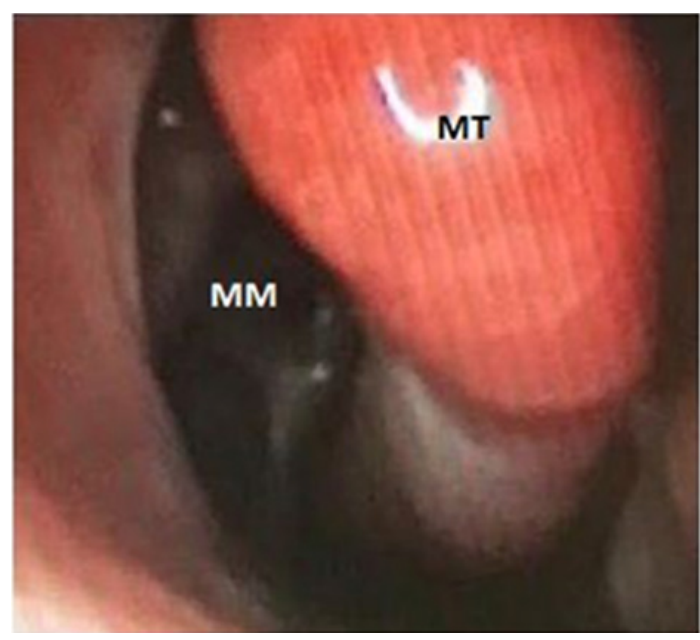

Figure IB Patency of the right middle meatus.

(MT: Middle Turbinate; MM: Middle Meatus; LNW: Lateral Nasal Wall)

Endoscopic Conchopexy Suture is another technique suggested by several authors as the most definitive method to prevent lateralization with $90-92 \%$ success rate ${ }^{3}$ (Figure 3). It is used a 4-0 resorbable suture (eg. Vicryl ${ }^{\mathrm{TM}}$, Polisorb ${ }^{\mathrm{TM}} \ldots$...) with the needle partially straightened that allows easy passage through the tissue.

The needle is introduced into the left nasal cavity oriented vertically, visualized with a $0^{\circ}$ nasal telescope, and passed through the head of the middle turbinate, septum and the contralateral middle turbinate in a single pass. It is then brought back to the right middle turbinate and passed through the septum to the initial side to be knotted with direct visualization. This technique has been criticized for its likely 
effect on olfaction but there are studies which confirm that it does not impair the function of olfaction. ${ }^{3}$ Endoscopic Conchopexy Suture has also been considered a difficult technical and lengthens the operating time due to the need to tie inside the nasal cavity, but a new technique has simplified this process of suture to avoid knot-tying within the nasal cavity using a monofilament barbed suture with knot tied. ${ }^{1}$ This technique is mainly indicated to cases of Endoscopic Surgery with an unstable middle turbinate and also if a septoplasty ES performed. In these cases the Bolgerization technique can produce major injure of the middle turbinate and a septal perforation, hence it is preferred to perform a conchopexy suture avoiding these complications. Moreover, Conchopexy Suture is more comfortable for patients because it needs no postoperative removable nasal packing.

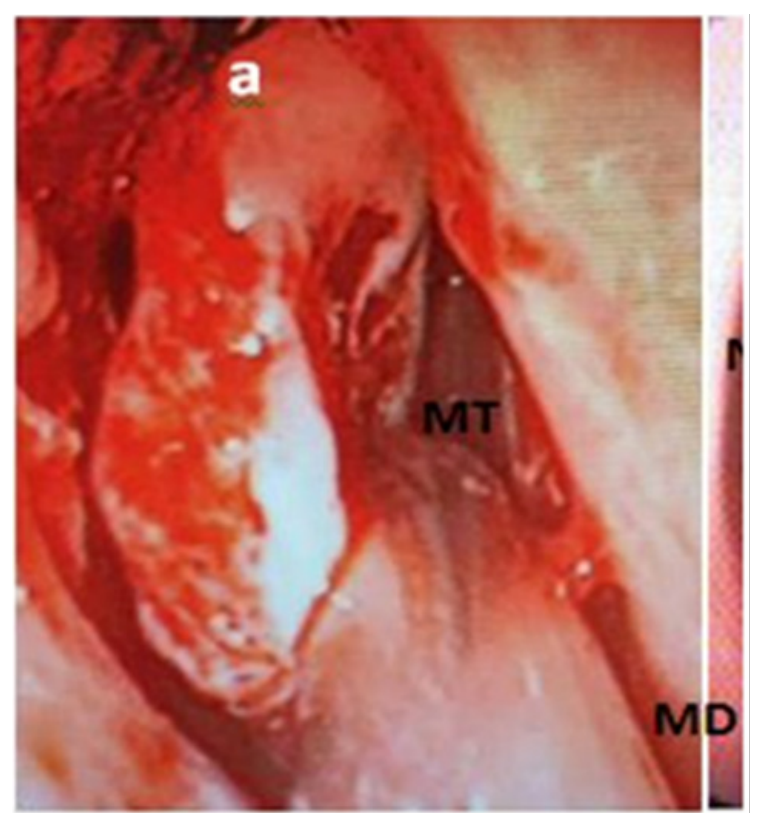

Figure 2A Bolgerization - Technique with microdebrider.

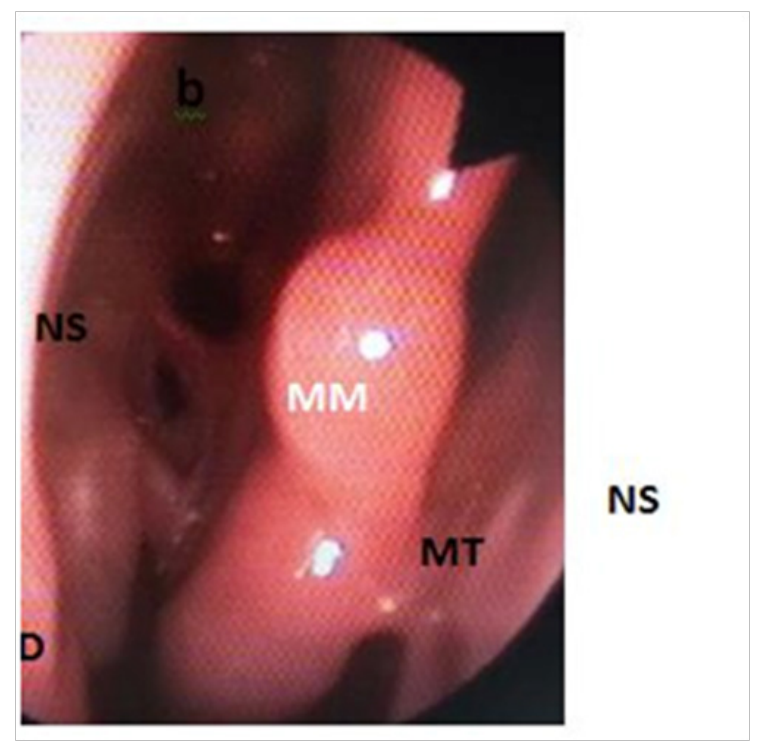

Figure 2B Bolgerization - Postoperative endoscopic view lyear after.

(MT: Middle Turbinate; MM: Middle Meatus; NS: Nasal Septum; MD: Microdebrider).

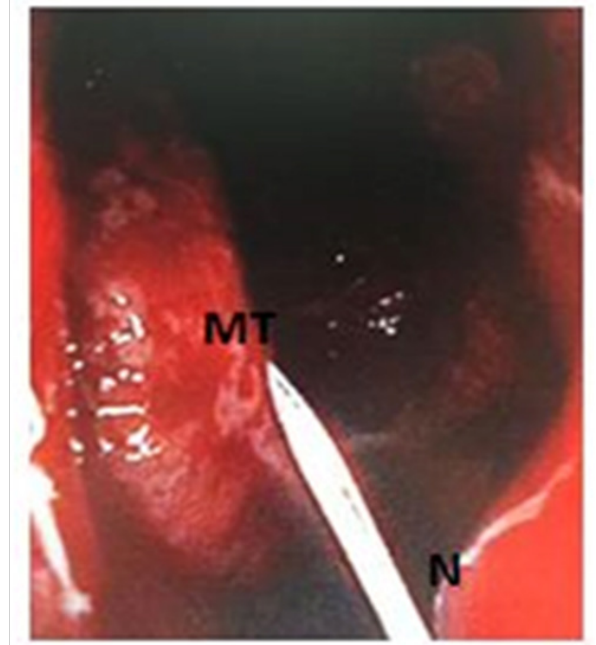

Figure 3A Conchopexy suture - Needle through the head of the middle turbinate.

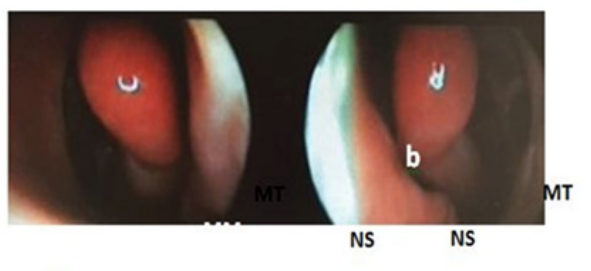

Figure 3B Conchopexy suture - Postoperative bilateral endoscopic view lyear after.

(MT: Middle Turbinate; MM: Middle Meatus; NS: Nasal Septum; N: Needle)

\section{Acknowledgments}

None.

\section{Conflicts of interest}

Author declares there are no conflicts of interest.

\section{Funding}

None.

\section{References}

1. Hudson S, Orlandi R. Knot-free suture medialization of the middle turbinate. Int Forum Allergy Rhinol. 2013;3(10):855-856.

2. Chen W, Wang Y, Bi Y, et al. Turbinate-septal suture for middle turbinate medialization: a prospective randomized trial. Laryngoscope. 2015;125(1):33-35.

3. Dutton JM, Hinton MJ. Middle turbinate suture conchopexy during endoscopic sinus surgery does not impair olfaction. Am J Rhinol Allergy. 2011;25(2):125-127.

4. Bolger WE, Kuhn FA, Kennedy DW. Middle turbinate stabilization after functional endoscopic sinus surgery: the controlled synechiae technique. Laryngoscope. 1999;109(11):1852-1853. 